\title{
Morphology Transformation of a Polymeric Micelle Induced by Two Encapsulated Particles
}

\author{
Q.-Y. ZHANG*AND X. XIANG \\ Department of Mathematics and Physics, Chongqing University of Science and Technology, \\ Chongqing, 401331, China
}

(Received April 5, 2014; in final form October 15, 2014)

\begin{abstract}
We begin with a pair of spherical particles encapsulated in a micelle and solve the problem within the selfconsistent field theory to investigate the morphology transformation of the micelle induced by two nano-particles. The micelles are based on amphiphilic diblock copolymer AB in homopolymer A solvents. The nano-particles are simple models of proteins or colloids. We predict different micelle structures, and relate these morphologies to particle sizes, distances between two particles, and chemical potentials of the amphiphile molecules. There are found complex structures of the micelle induced by the twin particles: many reverse micelles only with even numbers or only one micelle attached at one of the two particles. These results are of immediate interests to the interactions of micelles and particles, as well as to the transitions between vesicles and micelles.
\end{abstract}

DOI: 10.12693/APhysPolA.126.1338

PACS: 82.35.Np, 82.35.Jk

\section{Introduction}

Many amphiphilic molecules, such as surfactants and block copolymers, aggregate into micelles. Micelles selfassembled by amphiphilic molecules play an important role in many practical applications, such as templates for the synthesis of mesoporous materials, the transport of water-insoluble solutes, drug delivery [1-3], dragreducing agents [4], pharmaceutical applications [5], and gene delivery $[6,7]$. The interactions between micelles and particles [8] are not only of a fundamental scientific interest but play an extremely important role in many technological applications.

The interactions of nanoparticles with biological membranes have recently gained much interests among scientists in the field of nanotechnology [9-11]. As one of the major applications, nanoparticles can be designed to serve for drug delivery purposes by entering the target cell membrane and acting as drug containers [12]. The wrapping and internalization of nanoparticles by biomembranes plays a critical role in drug delivery applications and nanomedicine. Therefore it is crucial to understand the mechanisms by which nanoparticles interact with biological cells and enter the cell membrane.

Other example is the endocytosis. Endocytosis is a fundamental cellular process through which many artificial and natural biological objects are engulfed and internalized by the cell $[13,14]$. Wrapping and cellular uptake of technologically designed nanoparticles provides an important route to transport drug containers and deliver the cargo within the target cell. Cellular uptake of nanoparticles is associated with deformations induced on the plasma membrane of the cell by the particle.

\footnotetext{
*corresponding author; e-mail: qyzhang520@163.com
}

While there are several simulations [15-22] and theoretical studies [23-29] about the interactions of a single nanoparticle with a biomembrane, a comprehensive investigation on the effect of more than one particle is still missing. When more than one particle are adsorbed on a membrane, the interactions between the micelle and particles become much more complicated. The geometry of the membrane is strongly affected by the embedded particles. For a pair of particles encapsulated in a membrane, the shape deformations of the membrane depend on the relative distance between these particles.

Particularly, self-consistent theory is a powerful method taking into account entropy and enthalpic interaction. Our theoretical understanding can be built based on this well-tested model, which in many ways has provided useful insight into the fusion and fission of a membrane [30-32].

Despite all the studies in these research fields, it is still necessary for detailed understanding about the interactions of nanoparticles with micelles. With this paper, we attempt to bring some ideas and conclusions that might be useful on the interactions of micelles and particles.

\section{Model and calculation details}

The system consists of a copolymer-homopolymer mixture and two identical spherical nanoparticles. The incompressible mixture contains $n_{\mathrm{a}}$ amphiphilic diblock copolymers, composed of A (hydrophilic) and B (hydrophobic) monomers, and $n_{\mathrm{s}}$ solvent molecules, represented by hydrophilic homopolymers consisting of A segments only. All the segments are defined based on a common segment volume $\rho_{0}^{-1}$ and statical length $b$. All polymers are composed of $N$ segments. A fraction $f$ forms the hydrophilic block in the diblock. The interaction between hydrophobic and hydrophilic monomers is controlled by a local repulsion of strength $\chi$, the usual Flory-Huggins parameter, with a choice $\chi N=30$ here. 
The surface of the particle is hard and impenetrable. The total segment density in the vicinity of its surface drops to zero in a boundary region of width $\epsilon$ according to $[33-35]$ :

$$
\phi_{0}(r)= \begin{cases}0, & r \leq R, \\ \frac{1}{2} 1-\cos \left(\frac{\pi(r-R)}{\epsilon}\right), & R \leq r \leq R+\epsilon, \\ 1, & \text { else. }\end{cases}
$$

where $\epsilon$ is chosen much smaller than $R_{\mathrm{g}}$, the polymer radius of gyration.

For simplicity, the nanoparticle is modeled with a neutral surface, not hydrophilic or hydrophobic.

Within the well established SCFT [36-39], the boundary conditions are needed. For the four boundaries of our simulation box, the Neumann condition is used. At the boundary on the particle, the Dirichlet condition is used, because of the hard particle surface and polymer cannot cross the boundary. In grand canonical ensemble [40-42], the free-energy functional has the form

$$
\begin{aligned}
& \frac{N F}{\rho_{0} k_{\mathrm{B}} T V}=-\frac{1}{V} Q_{\mathrm{s}}-\frac{\exp \left(\mu / k_{\mathrm{B}} T\right)}{V} Q_{\mathrm{a}} \\
& \quad+\frac{1}{V} \int \mathrm{d} \boldsymbol{r} \chi N\left[\phi_{\mathrm{t}}(\boldsymbol{r}) \phi_{\mathrm{h}}(\boldsymbol{r})+\phi_{\mathrm{s}}(\boldsymbol{r}) \phi_{\mathrm{t}}(\boldsymbol{r})\right] \\
& +\frac{1}{V} \int \mathrm{d} \boldsymbol{r} H(\boldsymbol{r})\left[\phi_{\mathrm{h}}(\boldsymbol{r})+\phi_{\mathrm{s}}(\boldsymbol{r})-\phi_{\mathrm{t}}(\boldsymbol{r})\right] \\
& \quad-\frac{1}{V} \int \mathrm{d} \boldsymbol{r}\left[w_{\mathrm{h}}(\boldsymbol{r}) \phi_{\mathrm{h}}(\boldsymbol{r})+w_{\mathrm{t}}(\boldsymbol{r}) \phi_{\mathrm{t}}(\boldsymbol{r})+w_{\mathrm{s}}(\boldsymbol{r}) \phi_{\mathrm{s}}(\boldsymbol{r})\right] \\
& +\frac{1}{V} \int \mathrm{d} \boldsymbol{r} \xi(\boldsymbol{r})\left[\phi_{\mathrm{h}}(\boldsymbol{r})+\phi_{\mathrm{t}}(\boldsymbol{r})+\phi_{\mathrm{s}}(\boldsymbol{r})-\phi_{0}(\boldsymbol{r})\right] .
\end{aligned}
$$

Here $\mu$ is the relative chemical potential of amphiphile. Because of assumed incompressibility constraint, there is only one independent chemical potential. The local volume fractions of A (head) and B (tail) segments of the amphiphile are given by $\phi_{\mathrm{H}}(\boldsymbol{r})$ and $\phi_{\mathrm{T}}(\boldsymbol{r})$. Likewise, the $\phi_{S}(\boldsymbol{r})$ is the local concentration of solvent. We let $w_{\mathrm{H}}(\boldsymbol{r})$ denote the value of the mean field felt by head segments at $\boldsymbol{r}, w_{\mathrm{T}}(\boldsymbol{r})$ the field for tail segments, and $w_{\mathrm{S}}(\boldsymbol{r})$ the field for solvent segments. The $Q_{\mathrm{s}}$ and $Q_{\mathrm{a}}$ are the single chain partition functions of the solvent and amphiphile molecules subjected to the above fields. A Lagrange multiplier field $\xi(\boldsymbol{r})$ is introduced to enforce incompressibility. To form the starting configuration, an equilibrated spherical "micelle" is created by artificially "biased" structure with two identical particles locating close in the center of the micelle [30, 43-46]. All the figures of micelles in the paper are with the same size $60 \times 20 R_{\mathrm{g}}$. The radius of the particle is $R$.

\section{Results}

The shapes of the micelle including particles have complex geometries. We choose 4 representative systems, which predict the 4 probable morphology transformations of the micelle in their interactions with twin enclosed particles. The parameters of the 4 systems are shown in Table.

The total free energies of the micelles vary with the relative distances $d$ between nanoparticles, where $d$ is the separation between centers of the two spherical particles.

\section{TABLE}

The main parameters used in our calculations for the 4 systems.

\begin{tabular}{c|c|c|c}
\hline \hline System & $f$ & $\mu / k_{\mathrm{B}} T$ & $R$ \\
\hline I & 0.2 & 70 & 0.5 \\
II & 0.2 & 70 & 1.0 \\
III & 0.25 & 70 & 1.0 \\
IV & 0.2 & 60 & 1.0
\end{tabular}

The free energies of the 4 different systems in Table are shown in Figs. 1-5. The corresponding critical shape transformations of micelles induced by the two particles are also shown following each free energy curve. First we demonstrate the shape deformations of micelles in Fig. 1 for system I. Initially, the distance $d$ is small in Fig. 1a, the two particles are almost at the center of the micelle. It exhibits a clear micellar structure with a radius of approximately $7.1 R_{\mathrm{g}}$ when the head groups are included, corresponds to micelle with a diameter of $31 \mathrm{~nm}$, which is in the range of the experimental results [47]. Then, the two particles "move" apart from each other sufficiently slowly so that the micelle is maintained in quasi-equilibrium. At each given value of distance $d$, we calculate the free energy and the concentration distribution of the micelle. The free energy curve shows a straight line and increases monotonically with increasing distance $d$. As the distance is increasing, the original spherical micelle is stretched and lengthened gradually into a flat state.

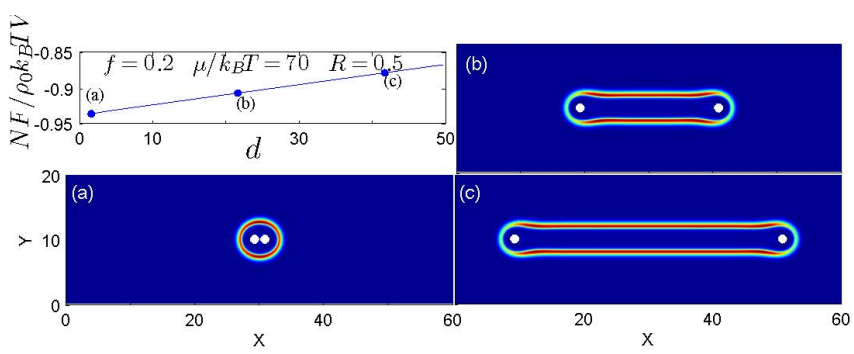

Fig. 1. System I. The free energy of micelle with $f=$ 0.2 and $\mu / k_{\mathrm{B}} T=70$, as a function of the distance $d$ between two equal-sized particles with radius $R=0.5$. The marks (a), (b) and (c) denote 3 representative states of the micelle, whose different morphologies are shown in the corresponding subfigures. Parts (a), (b) and (c) show the density distributions of the head parts of the amphiphiles, $\phi_{\mathrm{h}}$, corresponding to the 3 states marked in the free energy curve. The small white circles schematically denote the twin particles.

The most interesting phenomenon is the system II in Fig. 5. The free energy is shown in Fig. 2. The free energy curve demonstrates increasing zigzag shape with several sudden changes as the distance becomes large. In Fig. 5, the original spherical micelle in (1) forms a short lamellar inverted micelle between two particles in (2), which is stretched into two small spherical reverse 


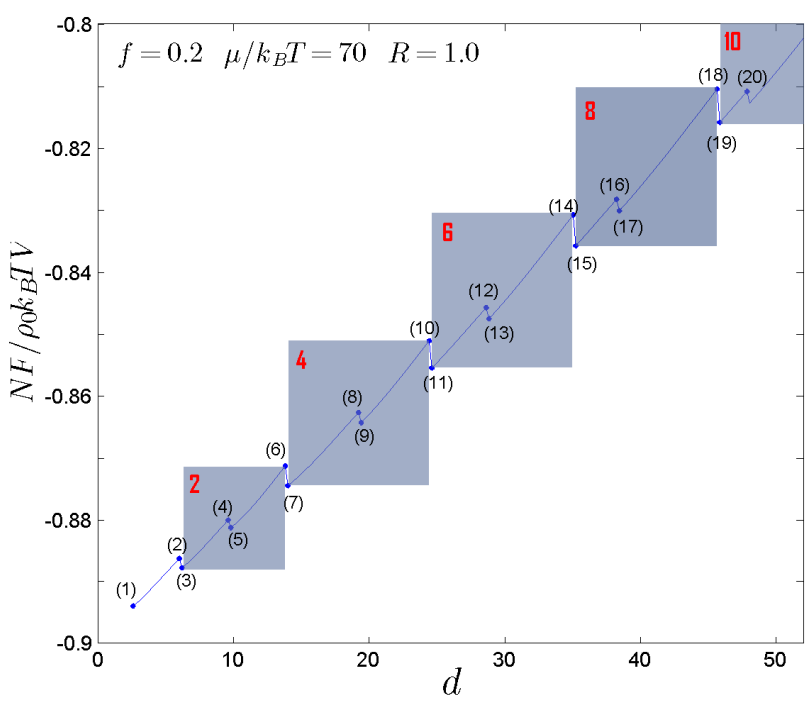

Fig. 2. System II. The free energy of micelle with $f=0.2$ and $\mu / k_{\mathrm{B}} T=70$, as a function of the distance $d$ between two equal-sized particles with radius $R=1.0$. The marks from (1) to (20) denotes the 20 critical states of the micelle, whose different morphologies will be shown in the Fig. 5 . The 5 rectangular shaded regions show the different parameter ranges in the energydistance plane, which correspondingly denote $2,4,6,8$ and 10 reverse micelles enclosed by the outer monolayer in Fig. 5.

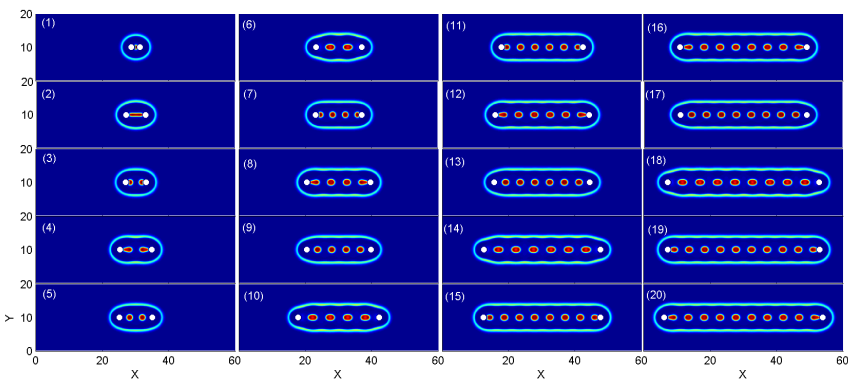

Fig. 3. System II. The density distributions of the head part of the amphiphiles, $\phi_{\mathrm{h}}$, corresponding to the 20 states marked in the free energy curve in Fig. 2. The system parameters are: $f=0.2, \mu / k_{\mathrm{B}} T=70$ and $R=1.0$. The small white circles schematically denote the twin particles.

micelles attached to the two particles in (3). When $d$ is further increased, the two small reverse micelles grow larger and larger gradually in (4), (5) and (6). Correspondingly in the free energy curve of Fig. 2, the shaded area enclosing marks from (3) to (6) shows the energydistance parameter range for the specific 2 inverted micelles issue. Then, with the increasing distance, 2 inverted micelles are stretched into 4 , and 4 into 6,6 into 8 in Fig. 5 (15), etc.

The inverted micellar structures are possible relevant to biomembrane processes, particularly to membrane fusion and exocytosis [48].

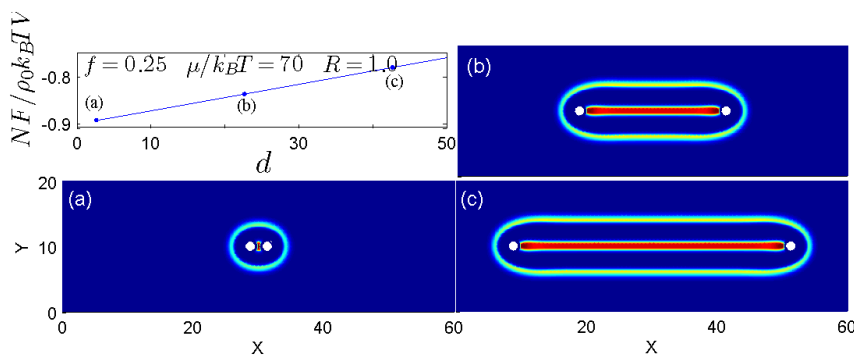

Fig. 4. (color online) System III. The free energy of micelle with $f=0.25$ and $\mu / k_{\mathrm{B}} T=70$, as a function of the distance $d$ between two equal-sized particles with radius $R=1.0$. The marks (a), (b) and (c) denotes 3 representative states of the micelle, whose different morphologies are shown in the corresponding subfigures. Figures (a), (b) and (c) show the density distributions of the head parts of the amphiphiles, $\phi_{\mathrm{h}}$, corresponding to the 3 states marked in the free energy curve. The small white circles schematically denote the twin particles.

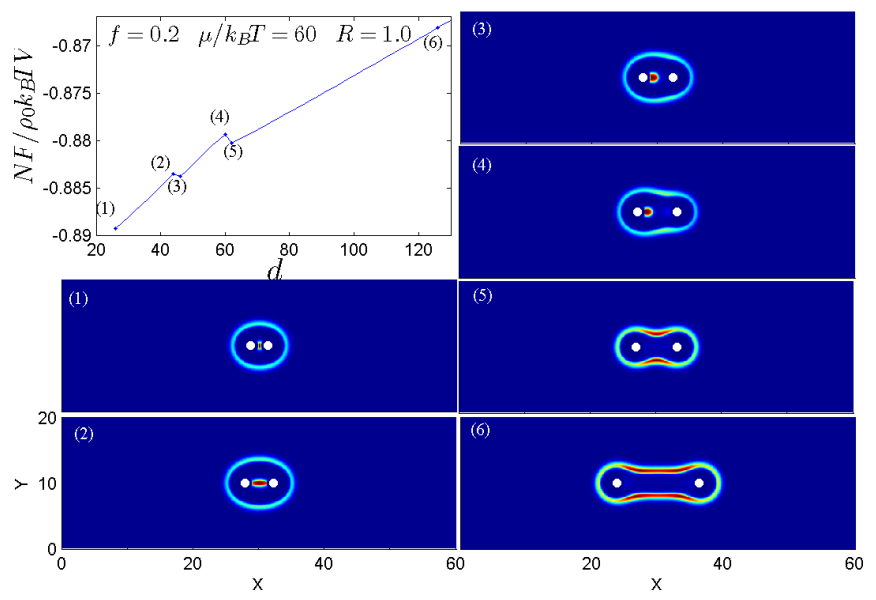

Fig. 5. System IV. The free energy of micelle with $f=$ 0.2 and $\mu / k_{\mathrm{B}} T=60$, as a function of the distance $d$ between two equal-sized particles with radius $R=1.0$. The marks from (1) to (6) denote 6 critical states of the micelle, whose different morphologies are shown in the corresponding subfigures. The 6 figures from (1) to (6) show the density distributions of the head parts of the amphiphiles, $\phi_{\mathrm{h}}$, corresponding to the 6 states marked in the free energy curve. The small white circles schematically denote the twin particles.

If the hydrophilic part is increased, such as $f=0.25$ in system III compared to system II, the inner inverted micelles between two particles do not be split, but be stretched to become long straight reverse micelles in Figs. $3 \mathrm{~b}$ and $\mathrm{c}$.

If the chemical potential is decreased such as $\mu / k_{\mathrm{B}} T=$ 60 in system IV compared to system III, the reverse micelle formed between two particles is only attached on one of the twin nanoparticles, shown in Figs. 4 (3) and (4).

If the hydrophilic part is increased such as $f=0.5$, for $\mu / k_{\mathrm{B}} T=70$ and $R=1.0$, the morphology of the micelle is similar to Fig. 1 of system I, but with dumbbell shape of micelle for large enough distance. 
For a larger particle, such as $R=1.5$, the inverted micelles between two particles are very complex, which are also examined in our calculations.

For the interested interactions between the micelle and the two encapsulated particles, only the three parameters $f, \mu / k_{\mathrm{B}} T$ and $R$ are changed. More parameters will not provide any new micelle shape transformations.

From the above free energy, it is a straightforward matter to evaluate the stretching force of the micelle. Such a force is the negative first derivative of the free energy $F$, with respect to the distance $d$. For example, the stretching force of system I in Fig. 1 is about $5 \rho_{0} b k_{\mathrm{B}} T$. Based on the typical value in Ref. [49], the force is in the order of $\mathrm{nN}$, which is in the range of Ref. [50].

\section{Conclusions}

We have reported on the morphology transformations of a micelle induced by two identical embedded particles, when the distance between two particles is increasing. For our parameters, the inner reverted micelles formed between the twin nanoparticles show very complex and interesting shapes, such as reverse micelles only with even numbers; only one inverted micelle is attached on one of the two particles. For particles with larger radii, the micelle may be stretched to form more complex structures.

It should be interesting to examine the influences of other parameters on the morphology transformations and the free energies, for example, the multicomponent effect of the amphiphiles, as well as the different length effect of the amphiphiles and solvents chains. Here, the particle is with neutral surfaces. Other parameters that may be considered is the adsorption strength between particle and amphiphile chains.

The self-consistent field theory in the grand canonical ensemble has the potential to dramatically accelerate the convergence of calculation [51]. Here, we work with an open system-grand canonical ensemble, where the polymer solution in volume $V$ and at temperature $T$ is connected to an external reservoir. For the gradually increasing distance $d$ between two particles, the percentages of each polymer species in the micelle system are always allowed to exchange particles with an infinite bulk according to the corresponding chemical potentials. In a relatively small micelle solution system, it seems like that the micelle is infinitely stretchable, which is certainly impossible. Actually, for a micelle with a certain size, the separation $d$ has a maximal value before the micelle completely ruptures. We will carry on this issue to enlarge the system and study this problem in canonical ensemble.

In our theoretical calculations, the particles are fixed. In experiments, the widely used optical tweezers can stable trap nanometre-sized objects [52,53]. Recently, the electrokinetic feedback guided by tracking of a fluorescent label in a fluidic slit with appropriately tailored topography [54] can trap and levitate gold particles, polymer beads and lipid vesicles with diameters of tens of nanometres for up to several hours. It is expected that our calculation results provide some theoretical information on the interactions of micelles with nanoparticles, and of course shed light on the regimes of the transformation between micelles and vesicles. Additional experiments could be performed to manipulate the nanoparticles and probe the interactions between nanoparticles and micelles.

\section{Acknowledgments}

This work is supported by the National Natural Science Foundation of China (Grant No. 20804060) and Research Foundation of Chongqing University of Science and Technology (Project No. CK2013B16).

\section{References}

[1] A. Blanazs, S.P. Armes, A.J. Ryan, Macromol. Rapid Commun. 30, 267 (2009).

[2] G. Gaucher, M.-H. Dufresne, V.P. Sant, N. Kang, J. Controlled Release 109, 169 (2005).

[3] Z. Luo, J. Jiang, J. Controlled Release 162, 185 (2012).

[4] J. Yang, Curr. Opin. Coll. Interface Sci. 7, 276 (2002).

[5] H. Nishi, J. Chromatogr. A 780, 243 (1997).

[6] J. Wen, H.-Q. Mao, W. Li, K.Y. Lin, J. Pharm. Sci. 93, 2142 (2004).

[7] S.-F. Chang, H.-Y. Chang, Y.-C. Tong, S.-H. Chen, Hum. Gene Ther. 15, 481 (2004).

[8] X.C. Qin, C.L. Ren, Chin. J. Polym. Sci. 30, 164 (2012).

[9] A.E. Nel, L. Madler, D. Velegol, T. Xia, Nature Mater. 8, 543 (2009).

[10] Y. Natsume, O. Pravaz, H. Yoshida, M. Imai, Soft Matter 6, 5359 (2010).

[11] H. Zhou, B.B. Gabilondo, W. Losert, W.W. van de Water, Phys. Rev. E 83, 011905 (2011).

[12] W.H.D. Jong, P.J. Borm, Int. J. Nanomed. 3, 133 (2008).

[13] I. Canton, G. Battaglia, Chem. Soc. Rev. 41, 2718 (2012).

[14] S.B. Sieczkarski, G.R. Whittaker, J. Gen. Virol. 83, 1535 (2002).

[15] H. Noguchi, M. Takasu, Biophys. J. 83, 299 (2002).

[16] L. Yang, G. Ning, J. Phys. Chem. B 114, 2749 (2010).

[17] R. Vacha, D. Frenkel, Nano Lett. 11, 5391 (2011).

[18] H. Ding, W. Tian, Y. Ma, ACS Nano 6, 1230 (2012).

[19] H. Ding, Y. Ma, Nanoscale 4, 1116 (2012).

[20] H. Ding, Y. Ma, Sci. Rep. 3, 2804 (2013).

[21] H. Ding, Y. Ma, Biomaterials 34, 8401 (2013).

[22] K. Yang, B. Yuan, Y. Ma, Nanoscale 5, 7998 (2013).

[23] R. Lipowsky, H.G. Dobereiner, Europhys. Lett. 43, 219 (1998).

[24] M. Deserno, Phys. Rev. E 69, 031903 (2004). 
[25] W.T. Gozdz, Langmuir 23, 5665 (2007).

[26] X. Yi, X. Shi, H. Gao, Phys. Rev. Lett. 107, 098101 (2011).

[27] S. Cao, G. Wei, J.Z.Y. Chen, Phys. Rev. E 84, 050901 (2011).

[28] A.H. Bahrami, Soft Matter 9, 8642 (2013).

[29] A.H. Bahrami, R. Lipowsky, T.R. Weikl, Phys. Rev. Lett. 109, 188102 (2012).

[30] K. Katsov, M. Müller, M. Schick, Biophys. J. 87, 3277 (2004).

[31] K. Katsov, M. Müller, M. Schick, Biophys. J. 90, 915 (2006).

[32] J.Y. Lee, M. Schick, Biophys. J. 92, 3938 (2007).

[33] M.W. Matsen, J. Chem. Phys. 106, 7781 (1997).

[34] J.Y. Lee, Z. Shou, A.C. Balazs, Phys. Rev. Lett. 91, 136103 (2003).

[35] M. Müller, K. Binder, J. Phys. Condens. Matter 17, S333 (2005).

[36] M.W. Matsen, M. Schick, Phys. Rev. Lett. 72, 2660 (1994).

[37] F. Schmid, J. Phys. Condens. Matter 10, 8105 (1998).

[38] M.W. Matsen, J. Phys. Condens. Matter 14, R21 (2002).

[39] Q.Y. Zhang, Chin. Phys. B 18, 0658 (2009).

[40] R.B. Thompson, M.W. Matsen, J. Chem. Phys. 112, 6863 (2000).
[41] K. Katsov, M. Müller, M. Schick, Biophys. J. 87, 3277 (2004)

[42] M.W. Matsen, J. Chem. Phys. 110, 4658 (1999).

[43] Q.Y. Zhang, Y.Q. Ma, J. Chem. Phys. 125, 164710 (2006).

[44] Q.Y. Zhang, Y.Q. Ma, J. Phys. Chem. B 110, 26279 (2006).

[45] J.Y. Lee, Z. Shou, A.C. Balazs, Phys. Rev. Lett. 91, 136103 (2003).

[46] Q.Y. Zhang, X. Xiang, Chin. Phys. B 22, 038201 (2013).

[47] G. Lei, K. Zhang, B. Peng, Y. Shi, Y. Chen, J. Polym. Sci. Part B. Polym. Phys. 50, 323 (2012).

[48] D.P. Siegel, Biophys. J. 49, 1155 (1986).

[49] E. Helfand, A.M. Sapse, J. Chem. Phys. 62, 1327 (1975).

[50] M.E. Solmaz, R. Biswas, S. Sankhagowit, J.R. Thompson, Biomed. Opt. Express 3, 2419 (2012).

[51] Z. Mester, N.A. Lynd, G.H. Fredrickson, Soft Matter 9, 11288 (2013).

[52] P.M. Bendix, L.B. Oddershede, Nano Lett. 11, 5431 (2011).

[53] C. Ghimire, D. Koirala, M.B. Mathis, E.E. Kooijman, H. Mao, Langmuir 30, 1370 (2014).

[54] M. Krishnan, N. Mojarad, P. Kukura, V. Sandoghdar, Nature 467, 692 (2010). 